\title{
Multimodal Image Registration by Information Fusion at Feature Level
}

\author{
Yang Li and Ragini Verma \\ Department of Radiology, University of Pennsylvania, Philadelphia, PA, USA, 19104 \\ \{Yang.Li, Ragini.Verma\}@uphs. upenn.edu
}

\begin{abstract}
This paper proposes a novel multimodal image registration method which can fully utilize the multimodal information and result in a more accurate unified deformation field. Different from the existing methods which fuse the information at the image/intensity level, the proposed method fuses the multimodal information at the feature level through Gabor wavelets transformation. At this level, complementary and redundant information is distinguished reliably and efficiently, and then combined and removed respectively. Experiments on both simulated and real T1+DTI image sets illustrate that the proposed method can effectively incorporate better characterization for white matter (WM) from the DTI and for gray matter (GM) from the T1 image and lead to a more accurate and efficient multimodal image registration which paves the way for the subsequent multimodal population-based studies.
\end{abstract}

\section{Introduction}

Clinical studies routinely acquire different magnetic resonance imaging (MRI) protocols such as structural images (T1, T2, FLAIR, mPRAGE ) and diffusion imaging such as Diffusion Tensor Imaging (DTI). Each of these modalities provides some unique characterization of tissue. For instance, structural images generate contrast between the gray matter (GM), white matter (WM) and cerebrospinal fluid (CSF). DTI captures the directional microstructural information within WM as a tensor, which complements the missing orientation information of structural images. Multimodal image registration has become an active research topic, because 1) it combines complementary information from different modalities and 2) the deformation field obtained is common to all the modalities. When the spatially normalized images are used for statistics, using the same deformation field makes them more comparable than using separate registrations for each of the modality which usually generates inconsistent deformation fields. It is also efficient, as registrations on all modalities are produced at the same time. In 1], Park et al proposed a deformable multimodal image registration method using multichannel demons [2, in which T2 and DTI were registered as a vector image. In this straightforward method, although every image channel is incorporated, they are assigned equal importance in the image matching. Or in other words, there is no separation between complementary and redundant information. More recently, in [3], multimodal image consisting of T1, DTI and 
cerebral blood flow $(\mathrm{CBF})$ were registered by defining the multimodal similarity metric as a weighted summation of each modality's similarity metric. Although spatially constant weights were used in the experiments, the author also suggested that a weighting function $\omega_{i}(\mathbf{x})$ defined on the image domain should generate better results. In Studholme's work 4, DTI was incorporated into the mapping between T1 as an constraint. Multichannel mutual information (MI) was used to match the multimodal image. Due to the impractical number of histogram bins required by the multichannel MI of all 7 image channels (T1 and 6 tensor components), a simplification of only using two tensor components, $D_{x x}$ and $D_{y y}$, was adopted based on the fact that the different diffusion directions is relatively un-correlated. For other multimodal image with large number of modalities, multichannel MI remains computationally expensive.

The challenging problem in multimodal image registration is to fuse the multimodal information and find a unified deformation field that can be applied to spatially normalize every image channel. The meaning of 'fuse' is two-fold here, merging the complementary (to be more accurate) and removing the redundant (to be more efficient). The main drawback of dealing with the information fusion problem at image/intensity level is that, it is difficult to extract and distinguish between complementary and redundant information, because the stronger signal (higher intensity value) does not necessarily indicates a stronger characterization ability of one modality. This ability is proportional to the contrast the modality can provide. Inspired by the ideas of multiscale decomposition (MSD)based information fusion scheme [5], which have been successfully used in image fusion [6], in this paper, we propose a novel multimodal image registration algorithm which fuses the multimodal information at feature level. By designing a matching metric through Gabor wavelets transformation, the proposed registration method can incorporate and enhance complementary information while eliminating the redundancies between the different channels. Therefore, this leads to a more accurate registration at a much lower computational cost. In experiments, we apply the new algorithm to T1+DTI image and obtain more accurate registration than using either of the two modalities individually.

\section{Methods}

Before describing the proposed registration algorithm, we will illustrate the basic idea of MSD-based information fusion scheme through an example.

\subsection{MSD-Based Information Fusion}

An image I can be decomposed by discrete wavelets transform (DWT) into a series of wavelets coefficients $c_{k, l, m, n}^{\mathbf{I}}$, where $k, l$ and $(m, n)$ indicate the decomposition level, the frequency band and the position in a frequency band, respectively. Because $\left\|c_{k, l, m, n}^{\mathbf{I}}\right\|$ reflects the local energy of the output of the filter banks and is a good indicator of the characterization ability of each modality at the specific scale, frequency band and position, the simplest but efficient information fusion rule is the choose-max scheme [5]. That is, to fuse two image 


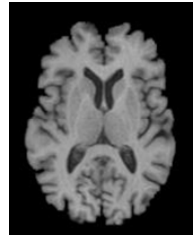

(a)

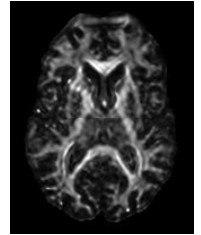

(b)

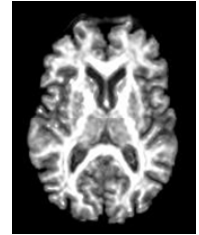

(c)

Fig. 1. An example of image fusion by the choose-max scheme. (a) T1 image; (b) FA image; (c) Fused image.

$\mathbf{X}$ and $\mathbf{Y}$, we can get the wavelets-based MSD representation of the fused image $\mathbf{Z}$ by only keeping the coefficient with the largest absolute value. After that, an inverse DWT can be performed to finally get the fused image $\mathbf{Z}$. As an example, we use a pair of co-registered T1 and fractional anisotropy (FA) [7] of the same subject to show the performance of the above fusion scheme. As shown in Fig. 1 the fused image gives good characterization on both WM and cortex which are not available in the input $\mathrm{T} 1$ and FA, individually.

\subsection{Choose-Max Metric for Matching}

Using the similar MSD-based Choose-Max information fusion scheme, we firstly define a dissimilarity metric for the multimodal image registration. However, since the objective of information fusion in registration is to fully utilize the multimodal information for a better matching criteria, but not to get a fused image explicitly, we use Gabor wavelets [8] for the multiscale analysis, instead of DWT. Gabor wavelet transformation has been shown to be optimal in the sense of minimizing the joint uncertainty in space and frequency, and has been widely used for feature extraction [9]. Although the registration is for 3D image, to alleviate the computational cost, we use 3 perpendicular (axial, coronal and sagittal) 2D Gabor filter banks to extract the features. A 2D Gabor filter can be viewed as a sinusoidal plane of particular frequency and orientation, modulated by a Gaussian envelope: $G(x, y)=s(x, y) g(x, y)$, where $s(x, y)$ is a complex sinusoid: $s(x, y)=\exp \left[-j 2 \pi\left(u_{0} x+v_{0} y\right)\right]$ and $g(x, y)$ is a 2D Gaussian envelope:

$$
g(x, y)=\frac{1}{\sqrt{2 \pi} \sigma_{x} \sigma_{y}} \exp \left[-\frac{1}{2}\left(\frac{x^{2}}{\sigma_{x}^{2}}+\frac{y^{2}}{\sigma_{y}^{2}}\right)\right]
$$

$\sigma_{x}$ and $\sigma_{y}$ characterize the spatial extent and bandwidth of $g$ along the respective axes, $u_{0}$ and $v_{0}$ are the shifting frequency parameters in the frequency domain. Using $G(x, y)$ as the mother wavelet, the Gabor wavelets, a class of self-similar functions can be obtained by appropriate dilations and rotations of $G(x, y)$ through: $G_{m, n}(x, y)=a^{-m} G\left(x^{\prime}, y^{\prime}\right)$, where $x^{\prime}=a^{-m}(x \cos \theta+y \sin \theta)$, $y^{\prime}=a^{-m}(x \sin \theta+y \cos \theta), a>1, \theta=n \pi / O, m=1 \cdots S n=1 \cdots O$. $O$ indicates the number of orientations and $S$ the number of scales in the multiresolution decomposition. These parameters can be set according to 9 to reduce the redundant information (caused by the nonorthogonality of the Gabor wavelets) in 
the filtered images. Given an image $I(x, y)$, the Gabor transform with orientation $n$ and scale $m$ can be computed as:

$$
F_{m, n}(x, y)=\int I\left(x_{1}, y_{1}\right) G_{m, n}^{*}\left(x-x_{1}, y-y_{1}\right) d x_{1} d y_{1}
$$

where $*$ indicates the complex conjugate.

Suppose we have two multimodal image $\mathbf{I}=\left[I_{1}, \cdots, I_{M}\right]$ and $\mathbf{J}=\left[J_{1}, \cdots, J_{M}\right]$, in which $I_{1}, \cdots, I_{M}$ and $J_{1}, \cdots, J_{M}$ are two collections of co-registered images generated by $M$ different modalities. The Gabor wavelet transformation of the $i$ th modality image $I_{i}$ and $J_{i}$ with orientation $n$ and scale $m$ are $F_{m n}^{I_{i}}$ and $F_{m n}^{J_{i}}$, respectively. Using the choose-max scheme, the dissimilarity between two voxel $\mathbf{I}\left(\mathbf{x}_{\mathbf{1}}\right)$ and $\mathbf{J}\left(\mathbf{x}_{\mathbf{2}}\right)$ can be measured as:

$$
D_{1}\left(\mathbf{I}\left(\mathbf{x}_{\mathbf{1}}\right), \mathbf{J}\left(\mathbf{x}_{\mathbf{2}}\right)\right)=\sqrt{\sum_{m=1}^{S} \sum_{n=1}^{O} \sum_{i=1}^{M} w_{m, n, i}\left(\left\|F_{m, n}^{I_{i}}\left(\mathbf{x}_{\mathbf{1}}\right)\right\|-\left\|F_{m, n}^{J_{i}}\left(\mathbf{x}_{\mathbf{2}}\right)\right\|\right)^{2}}
$$

where

$$
w_{m, n, i}= \begin{cases}1 & \text { if } i=\underset{j=1 \cdots M}{\operatorname{argmax}}\left\|F_{m, n}^{I_{j}}\left(\mathbf{x}_{\mathbf{1}}\right)\right\| \\ 0 & \text { otherwise }\end{cases}
$$

Using $D_{1}$ on two $\mathrm{T} 1+\mathrm{FA}$ image sets, the similarity between a reference point on the template and other points on a subject can be computed and shown in Fig. 2(b) as a similarity map.

\subsection{Max-Index Metric for Matching}

To utilize the information which is not included in (3) without too much computational cost, we can design another metric using the max indices which we already have. Let $p_{m, n}^{\mathbf{I}}(\mathbf{x})$ denote the label of the modality which generates the strongest Gabor feature at scale $m$ and orientation $n$ on $\mathbf{I}(\mathbf{x})$. Arrange these labels into a vector form $\mathbf{p}^{\mathbf{I}}(\mathbf{x})=\left[p_{1,1}^{\mathbf{I}}(\mathbf{x}), \cdots, p_{S, O}^{\mathbf{I}}(\mathbf{x})\right]$. Then $\mathbf{p}^{\mathbf{I}}(\mathbf{x})$ is a very informative fingerprint of voxel $\mathbf{I}(\mathbf{x})$. Let $L\left(\mathbf{I}\left(\mathbf{x}_{\mathbf{1}}\right), \mathbf{J}\left(\mathbf{x}_{\mathbf{2}}\right)\right)$ be the number of equal labels between $\mathbf{p}^{\mathbf{I}}\left(\mathbf{x}_{\mathbf{1}}\right)$ and $\mathbf{p}^{\mathbf{J}}\left(\mathbf{x}_{\mathbf{2}}\right)$. Thus, $L$ is a similarity metric with an increasing resolution when $S$ and $O$ increase. Here, the 3 perpendicular 2D Gabor filter banks each has 4 scales and 6 orientations. Therefore, $L$ has the ability to differentiate the brain tissue into $4 \times 6 \times 3=72$ classes. Fig. 2(c) shows the similarity map generated by metric $L$. As we can see, at this resolution level, only $L$ can give considerable discriminatory power. More importantly, this is also a clear evidence showing the correctness and effectiveness of the choose-max fusion rule. Because if it is not reliable, we can not get such a specific similarity map by using only $L$. By combining $L$ with (3), the metric for multimodal image matching is finally defined as:

$$
D\left(\mathbf{I}\left(\mathbf{x}_{\mathbf{1}}\right), \mathbf{J}\left(\mathbf{x}_{\mathbf{2}}\right)\right)=\left[1-\frac{L\left(\mathbf{I}\left(\mathbf{x}_{\mathbf{1}}\right), \mathbf{J}\left(\mathbf{x}_{\mathbf{2}}\right)\right)}{3 S O+1}\right] \cdot D_{1}\left(\mathbf{I}\left(\mathbf{x}_{\mathbf{1}}\right), \mathbf{J}\left(\mathbf{x}_{\mathbf{2}}\right)\right)
$$

The discriminative ability of metric $D$ is shown in Fig. $2(\mathrm{~d})$. 


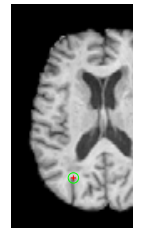

(a)

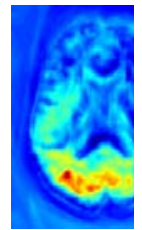

(b)

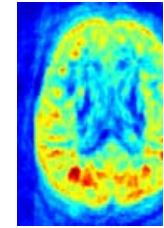

(c)

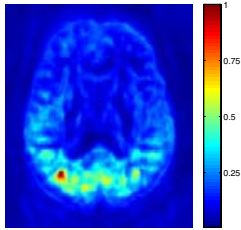

(d)

Fig. 2. Similarity map generated by different metrics. (a) shows the reference point (denoted by a red cross) on T1. (b) is the similarity map generated by $D_{1}$. (c) is the similarity map generated by metric $L$ (normalized to $\left[\begin{array}{ll}0 & 1\end{array}\right]$ ). (d) is the similarity map generated by metric $D$. Red indicates high similarity.

\subsection{Deformable Registration of Multimodal Image}

With the dissimilarity metric defined in (5), the problem of registering multimodal image $\mathbf{I}(\mathbf{x})$ to multimodal image $\mathbf{J}(\mathbf{x})$, can be defined as finding a optimal transformation $h(\mathbf{x})$ which minimizes

$\int_{\mathbf{x}_{\mathbf{1}} \in \Phi_{\mathbf{I}}} D\left(\mathbf{I}\left(\mathbf{x}_{\mathbf{1}}\right), \mathbf{J}\left(h\left(\mathbf{x}_{\mathbf{1}}\right)\right)\right) d \mathbf{x}_{\mathbf{1}}+\int_{\mathbf{x}_{\mathbf{2}} \in \Phi_{\mathbf{J}}} D\left(\mathbf{I}\left(\mathbf{x}_{\mathbf{1}}\right), \mathbf{J}\left(h^{-1}\left(\mathbf{x}_{\mathbf{2}}\right)\right)\right) d \mathbf{x}_{\mathbf{2}}+\alpha \int_{\mathbf{x}_{\mathbf{1}} \in \Phi_{\mathbf{I}}}\left\|\nabla^{2} h(\mathbf{x})\right\| d \mathbf{x}_{\mathbf{1}}$

By hierarchically optimizing (6) through the deformation model on the lines of the HAMMER algorithm [10, we develop a multimodal image registration algorithm. To select the active points (which drive the registration), we also adopt the choose-max criterion. After the edge map [11 of each channel is computed, a fused edge map is obtained using the choose-max scheme. On this fused map, the portion of points with higher edge intensity are selected as the active points. After that, in a hierarchical manner, by adding more and more active points to guide the deformation, this algorithm generates the final spatial transformation by concatenating the hierarchical sequence of piecewise smooth transformations obtained at each stage. Since DTI is one of the modalities in our experiments, the obtained deformation field is also used to determine the tensor reorientation by using a spatially adaptive procedure that estimates the underlying fiber orientation [12].

\section{$3 \quad$ Experiments and Results}

\subsection{Discriminatory Ability of the Metric}

The discriminatory ability of the proposed metric is firstly tested by comparing the similarity maps generated by different metrics. To make the comparison clear, we use multimodal image only consists of T1 and FA. In preprocessing, T1 is affinely registered to $B_{0}$ of DTI and re-sampled to have the same size $(256 \times 256 \times 70)$ and resolution $(0.9375 \times 0.9375 \times 2.5 \mathrm{~mm})$. The Gabor filter bank is set to have 6 orientations and 4 scales. Three other metrics are used 


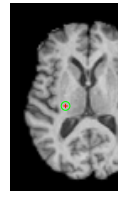

(a)

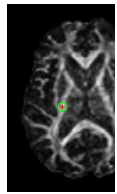

(b)

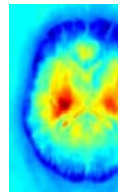

(c)

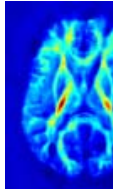

(d)

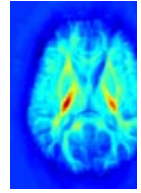

(e)

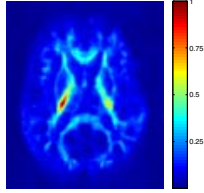

(f)

Fig. 3. Comparison of the similarity maps of a reference point on WM. (a) and (b) show the reference point (denoted by a red cross) on T1 and FA, respectively; (c) and (d) are similarity map only using $\mathrm{T} 1$ and FA, respectively; (e) is the similarity map using both $\mathrm{T} 1$ and FA without fusing; (f) is the similarity map given by the new metric defined in (5).

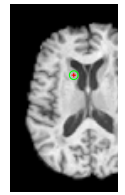

(a)

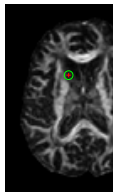

(b)

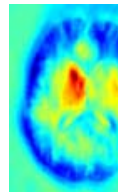

(c)

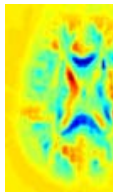

(d)

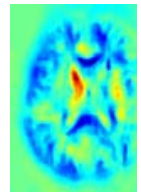

(e)

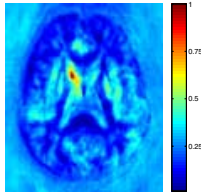

(f)

Fig. 4. Comparison of the similarity maps of a reference point on GM. Legends are the same as those in Fig. 3

for comparison: metric only using the Gabor features from T1 or FA and the metric using features from both $\mathrm{T} 1$ and FA but without fusing (as in (3) with $\left.w_{m, n, i}=1, \forall m, n, i\right)$. As expected, for a reference point on WM, the metric only using FA features (Fig. $3(\mathrm{~d})$ ) is more discriminative than the metric only using T1 features (Fig. 3(c) ). And similarly, the metric only using T1 features (Fig. 4(c) is more discriminative on GM than the metric only using FA features (Fig. 4(d) . Although for reference point on GM, the metric using both T1 and FA without fusing improves the discriminatory power (Fig. 4(e), by comparing Fig. 3(e) with Fig. 3(d), we also found that this metric actually degenerates the discriminatory ability on WM. In comparison, the proposed new metric (defined in (5) ) correctly utilizes the information from both channels and gives the most discriminative result on both WM (Fig. 3(f) and GM (Fig. 4(f) .

\subsection{Registration of Simulated and Real Images}

Ten simulated DTI+T1 image set are generated by applying ten simulated deformation fields on a template DTI+T1 image set (with the same size, resolution and preprocessing as used in Sect. 3.1). These ten simulated DTI+T1 image are then registered back to the template using the Gabor features extracted from T1 and 5 DTI-derived scalar images (FA, apparent diffusion coefficient (ADC) 7], $C_{s}$ (sphericity), $C_{p}$ (oblateness) and $C_{l}$ (prolateness) [13]). For comparison, 3 registrations were computed: the proposed method and the other two methods which only use features from one modality, either T1 or DTI. The deformation fields generated by the 3 methods are then compared with the simulated 


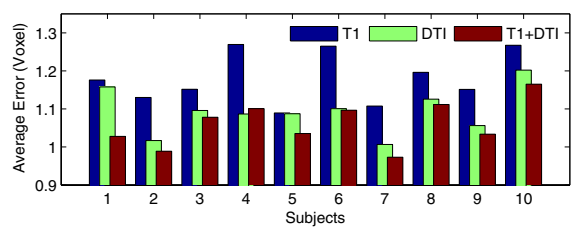

(a) Errors on deformation field

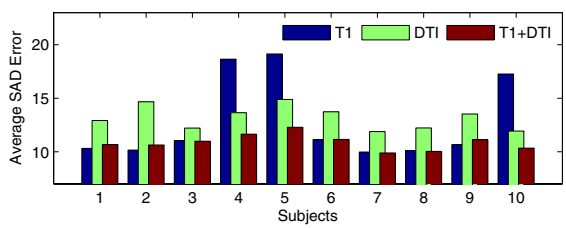

(c) Errors on registered T1

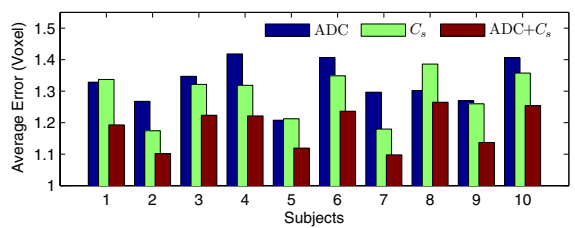

(b) Errors on deformation field

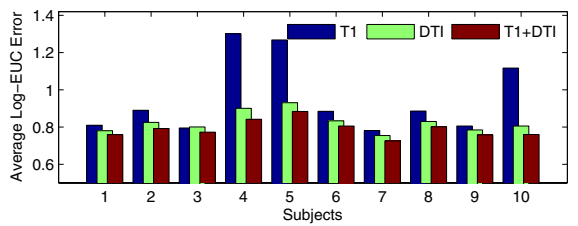

(d) Errors on registered DTI

Fig. 5. Comparison of the registration accuracy

deformation fields (ground truth). The results are summarized in Fig. 5(a), in which the proposed method, by utilizing the information from both modality, outperforms the other two methods on most of the subjects. The performances of the three methods are also compared on ten real T1+DTI images. After warping both T1 and DTI using the 3 deformation fields generated by the 3 methods, the registration accuracies were compared on both the registered $\mathrm{T} 1$ and the registered DTI based on the average Sum Absolute Difference (SAD) and LogEuclidean (Log-EUC) 14 metric, respectively. In both of these comparisons (as summarized in Fig. 5(c) and Fig. 5(d) , the proposed method generates the smallest error on most of the subjects, especially the 3 difficult subjects $(4,5$ and $10)$, on which the single modality methods generate relatively high registration error. This suggests that fusing the information from multi-modalities can help to robustly obtain accurate registration. We also found that, on some subjects (simulated subject 4 and real subject 1, 2 and 9), the proposed method does not give the best results (but difference is slight). One of the possible reasons that may cause registration error for the proposed method is the registration error between T1 and DTI in the preprocessing step. Therefore, to exclude the influence of this factor, another experiment was also conducted on the simulated subjects only using $\mathrm{ADC}$ and $C_{s}$ (they are inherently co-registered). As shown in Fig. 5(b), the proposed method consistently gives the best result in this experiment. And because ADC and $C_{s}$ have less redundant and more complementary information, the proposed method gains more improvement. In the future, more advanced inter-modality registration algorithm will be adopted to improve the intra-subject registration between T1 and DTI.

\section{Summary}

In this paper, we proposed a multimodal image registration framework based a new metric which combines complementary and removes redundant information 
from different modalities at the feature level. Experiments on both simulated and real multimodal images (DTI+T1) illustrate that the proposed method can effectively fuse the information from different modalities and result in a more accurate unified registration. In the future, we plan to explore more advanced fusion schemes of the Gabor features. As an application, we also plan to apply this method on clinical studies for joint comparative statistics on T1 and DTI.

Acknowledgments. The authors and the work was supported by the National Institute of Health via grant R01-MH-079938. We also acknowledge the help from the work of Dr. Dinggang Shen and Yangming Ou.

\section{References}

1. Park, H.J., Kubicki, M., Shenton, M.: Spatial normalization of diffusion tensor MRI using multiple channels. Neuroimage 20, 1995-2009 (2003)

2. Thirion, J.P.: Non-rigid matching using demons. In: Proceedings of IEEE Conference on Computer Vision and Pattern Recognition (CVPR), pp. 245-251 (1996)

3. Avants, B.B., Duda, J.T., Zhang, H., Gee, J.C.: Multivariate normalization with symmetric diffeomorphisms for multivariate studies. In: Ayache, N., Ourselin, S., Maeder, A. (eds.) MICCAI 2007, Part I. LNCS, vol. 4791, pp. 359-366. Springer, Heidelberg (2007)

4. Studholme, C.: Incorporating DTI data as a constraint in deformation tensor morphometry between T1 MR images. In: Information Processing in Medical Imaging (IPMI), pp. 223-232 (2008)

5. Zhang, Z., Blum, R.S.: A categorization of multiscale-decomposition-based image fusionschemes with a performance study for a digital camera application. Proceedings of the IEEE 87(8), 1315-1326 (1999)

6. Alfano, B., Ciampi, M., De Pietro, G.: A wavelet-based algorithm for multimodal medical image fusion. In: Semantic Multimedia, pp. 117-120 (2007)

7. Bassera, P.J., Pierpaoli, C.: Microstructural and physiological features of tissues elucidated by quantitative-diffusion-tensor MRI. Journal of Magnetic Resonance 111(3), 209-219 (1996)

8. Daugman, J.: Complete discrete 2 d gabor transforms by neural networks for image analysis and compression. IEEE Transactions on Acoustics 36(7), 1169-1179 (1988)

9. Ma, W., Manjunathi, B.: Texture features for browsing and retrieval of image data. IEEE Trans. Pattern Anal. Machine Intell. 18(8), 837-842 (1996)

10. Shen, D.: Image registration by local histogram matching. Pattern Recognition 40, 1161-1171 (2007)

11. Canny, J.: A computational approach to edge detection. IEEE Trans. Pattern Anal. Machine Intell. 8(6), 679-698 (1986)

12. Xu, D., Mori, S., Shen, D., Zijl, P.C.M.V., Davatzikos, C.: Spatial normalization of diffusion tensor fields. Magnetic Resonance in Medicine 50(1), 75-182 (2003)

13. Westin, C.F., Maier, S.E., Mamata, H., Nabavi, A., Jolesz, F.A., Kikinis, R.: Processing and visualization of diffusion tensor MRI. Medical Image Analysis 6(2), 93-108 (2002)

14. Arsigny, V., Fillard, P., Pennec, X., Ayache, N.: Log-euclidean metrics for fast and simple calculus on diffusion tensors. Magnetic Resonance in Medicine 56, 411-421 (2006) 